\title{
In-patient treatment in functional and sectorised care: patient satisfaction and length of stay
}

\author{
V.J. Bird, D. Giacco, P. Nicaise, A. Pfennig, A. Lasalvia, M. Welbel and S. Priebe
}

\section{Background}

Debate exists as to whether functional care, in which different psychiatrists are responsible for in- and out-patient care, leads to better in-patient treatment as compared with sectorised care, in which the same psychiatrist is responsible for care across settings.

\section{Aims}

To compare patient satisfaction with in-patient treatment and length of stay in functional and sectorised care.

\section{Method}

Patients with an ICD-10 diagnosis of psychotic, affective or anxiety/somatoform disorders consecutively admitted to an adult acute psychiatric ward in 23 hospitals across 11 National Health Service trusts in England were recruited. Patient satisfaction with in-patient care and length of stay (LOS) were compared (trial registration ISRCTN40256812).

\section{Results}

In total, 2709 patients were included, of which 1612 received functional and 1097 sectorised care. Patient satisfaction was significantly higher in sectorised care $(\beta=0.54,95 \% \mathrm{Cl} 0.35-0.73$, $P<0.001$ ). This difference remained significant when adjusting for locality and patient characteristics. LOS was 6.9 days shorter for patients in sectorised care $(\beta=-6.89,95 \% \mathrm{Cl}-11.76$ to -2.02 , $P<0.001$ ), but this difference did not remain significant when adjusting for clustering by hospital $(\beta=-4.89,95 \% \mathrm{Cl}-13.34$ to $3.56, P=0.26)$.

\section{Conclusions}

This is the first robust evidence that patient satisfaction with inpatient treatment is higher in sectorised care, whereas findings for LoS are less conclusive. If patient satisfaction is seen as a key criterion, sectorised care seems preferable.

\section{Declarations of interest}

None.

\section{Copyright and usage}

(c) The Royal College of Psychiatrists 2018
There is an ongoing debate about whether separate psychiatrists should be responsible for in- and out-patient care (functional care) or whether patients should be treated by the same psychiatrist across both settings (sectorised care). ${ }^{1-4}$ Over the past decade, various mental health services in the National Health Service (NHS) in England have moved away from the traditional sectorised forms of care, with the majority of the 58 mental health trusts now operating a functional split. It has been argued that these changes towards functional care, which were often costly from both a financial and resource perspective, would improve in-patient care. Yet, so far, there has been no high-quality evidence supporting this claim. ${ }^{1,2}$ This study aims to provide such evidence and compares two key indicators of the quality of in-patient treatment - patient satisfaction and length of stay $(\mathrm{LOS})$ - in functional and sectorised care.

\section{Method}

\section{Design}

Data from the present study is taken from 'Comparing policy framework, structure, effectiveness, and cost effectiveness of functional and integrated systems of mental health care study (COFI)' - a European Union study being conducted across five European countries (Belgium, England, Germany, Italy and Poland) (trial registration: ISRCTN40256812). COFI is a natural experiment assessing the outcomes of different ways of organising the link between in- and out-patient care. The full protocol for the COFI study has been previously published. ${ }^{5}$ For comparing satisfaction with in-patient care and LoS in functional and sectorised care, this paper focuses on the English site, as in England - unlike in other countries - the allocation of a patient to either functional or sectorised care is determined by location alone and not influenced by the characteristics of the patient.

\section{Setting}

A total of 23 hospitals were included in the study from 11 NHS trusts in England. In 11 hospitals run by five trusts (Camden and Islington NHS Foundation Trust, Manchester Health and Social Care Trust, North East London NHS Foundation Trust, Oxford Health NHS Foundation Trust and South Staffordshire, Shropshire and Shrewsbury NHS Foundation Trust) functional care was provided. In nine hospitals of five other trusts (East London NHS Foundation Trust, Cornwall Partnership Foundation Trust, Bradford District Care Trust, Dudley and Walsall Mental Health Partnership Trust and Pennine Care NHS Foundation Trust) sectorised care was provided. In one trust (North Essex Partnership Foundation Trust), both sectorised and functional care were routinely in operation, with functional care in two hospitals, and sectorised care in one other hospital.

\section{Eligibility criteria}

The hospitals included in the study were selected to represent a range of settings across England including urban, semi-urban and rural areas. To be included, the current form of care (i.e. sectorised or functional) needed to be in routine operation for at least 1 year and the hospital was required to have no plans to change the type of care they provided for the duration of the study. This meant that hospitals were excluded, even if run by a participating trust, if the current model of care had not been in place for at least 1 year, leading to the exclusion of three hospitals. All general adult psychiatric wards within an included hospital took part in the study, giving a total of 80 wards.

Patients were eligible if they met the following criteria: (i) a formal ICD-10 diagnosis ${ }^{6}$ (primary or secondary) of psychotic disorders (F2), affective disorders (F3) or anxiety/somatoform disorders (F4) or if they had a working diagnosis of F2-F4 as 
confirmed by the treating clinician; (ii) 18 years of age or older; (iii) admitted to a general adult psychiatric in-patient ward; (iv) capacity to provide informed consent; and (v) sufficient command of English to provide written informed consent and understand the questions in the research interviews. Patients with an organic brain disorder or too severe cognitive impairment to enable completion of the study measures were excluded. Additionally, patients who did not have a confirmed diagnosis at admission, and who did not go on to receive a confirmed F2-F4 diagnosis (at discharge) were excluded from the study, and no further data collected. No upper age limit was applied, providing the individual was recruited from an adult acute ward.

\section{Measures}

Outcomes for the study were mean satisfaction scores as rated on the Client Assessment of Treatment Scale (CAT) ${ }^{7}$ and length of hospital stay for the hospital admission for which they were included in the study. The CAT is a seven-item patient-reported outcome measure developed to assess satisfaction with in-patient care. ${ }^{7}$ The seven items included in the measure assess satisfaction with (i) treatment received; (ii) engagement with the psychiatrist; (iii) relations with other staff members; (iv) medication; (v) other elements of treatment; (vi) feeling respected; and (vii) whether the patient feels the treatment is right for them. All seven items are rated on a Likert scale ranging from 0 (not at all/very unpleasant) to 10 (yes entirely/very pleasant) and the overall mean score per item is calculated for the measure. ${ }^{7}$ The measure had been widely used with more than 4000 psychiatric in-patients ${ }^{8-11}$ and has been demonstrated to have good internal consistency ${ }^{10}$ and internal validity, ${ }^{9}$ as well as demonstrating factorial invariance and good factorial validity. ${ }^{12}$

Additionally, the following variables were collected for each patient: (i) sociodemographic characteristics; (ii) social situation including employment, accommodation, living situation, friendships, being in receipt of benefits; (iii) psychiatric and non-psychiatric diagnoses according to the ICD-10, with the primary clinical diagnosis at discharge established from the discharge summary and/or medical records; (iv) severity of illness as rated by the treating psychiatrist using the Clinical Global Impression Scale (CGI); ${ }^{13}$ and (v) admission details including date of admission and discharge, whether the current admission is the first one or not; and formal status at admission (involuntary/voluntary). Sociodemographic characteristics and social situation were completed by patients, and all clinical information was obtained from medical records.

\section{Procedure}

All new admissions to general adult psychiatric wards within the included hospitals were screened on every working day between 20 August 2014 and 31 December 2015. Individuals who met the inclusion criteria were initially approached by a member of the clinical team within 2 working days of admission. Where individuals did not have capacity to provide informed consent, they were reapproached in the following days. Trained researchers (from a psychology, nursing or psychiatry background) completed the assessment booklet with the participant. Medical records were used to collect clinical data such as date of admission and discharge, diagnosis at admission, primary diagnosis at discharge, legal status and additional physical health diagnoses. Following completion of the assessment, the patient's treating clinician was approached to complete the CGI measure regarding the patient's clinical status at admission. Each individual was only included in the study once, regardless of the number of admissions during the recruitment period. Patients were approached within 2 days of admission and attempts were made to assess patients within the first days to (i) avoid selection biases through early discharge; and (ii) have a consistent time frame rather than linking assessments to variable discharge dates. Ethical approval for the study was obtained from NRES Committee North East - Newcastle \& North Tyneside (ref: 14/NE/1017).

\section{Data analysis}

The mean for the CAT was calculated for each person. As data was collected at the time of recruitment, missing data were minimal. Where $20 \%$ of the data or less were missing for the CAT, the means of the non-missing items were used to replace missing values. LoS was defined as the number of nights the person was admitted calculated from the date of admission and date of discharge, which were always obtained from medical records. The mean LoS was calculated to allow for us to control for the effects of clustering at the hospital level and the impact of covariates within the analysis. The first quartile, second quartile (median) and third quartile LoS were also calculated because of the nonnormal distribution of the data and the potential outliners at the right end of the distribution. Additionally, Kaplan-Meier survival curves were drawn for functional and sectorised care.

Descriptive statistics for all sociodemographic, social and clinical variables were tabulated for the two forms of care and any differences between them assessed using $t$-tests (for continuous data) or chi-squared tests (for categorical data). Descriptive statistics were also calculated for the seven individual items on the CAT; however, we did not test for any significant differences between functional and sectorised care at the item level as the individual item scores as such have not been developed for this purpose. ${ }^{7}$

Mixed-effects linear regression with unstructured variance matrix was used to test for the impact of model of care (i.e. specialisation or functional) on the CAT. As per protocol, within the models, hospital was added as a random effect to adjust for clustering, whereby individuals recruited and receiving treatment from the same hospital were clustered together. The following covariates that were pre-specified in the data analysis plan were included as fixed effects: age, gender, severity of illness, first or repeat admission and legal status. Any variables shown to significantly differ at baseline between the two systems were included in the model. Given that LoS was not normally distributed, we conducted a quantile regression to test for significant differences in the first, second or third quartiles for LoS. The quantile regression used clustered standard error and the same covariates as applied within the regression model. Additionally, because of the large sample size, mixedeffects linear regression with unstructured matrix was used to confirm the findings of the quantile regression. As LoS represents duration data, a sensitivity analysis was conducted to analyse the data using a multilevel generalized linear model (GLM) with gamma distribution.

\section{Results}

A total of 8771 participants were screened within the study period. Of these, 6224 were eligible for inclusion and 3021 recruited, giving an opt-in rate of $48.5 \%$ (Fig. 1). Of the recruited participants, 312 individuals (10.3\%) were retrospectively excluded at the point of discharge as the working diagnosis of F2-F4 was not confirmed. In total, 2709 eligible individuals were recruited and included in the analysis, of which 1612 received functional and 1097 sectorised care.

Characteristics of the participants are shown in Table 1. The average age of participants was 40.6 years and $58.5 \%$ were male. There was a statistically significant difference in the level of education, with a lower proportion of individuals within sectorised sites having completed tertiary education and a greater proportion completing primary or secondary education compared with functional care. Additionally, the proportion of people born in the UK differed 


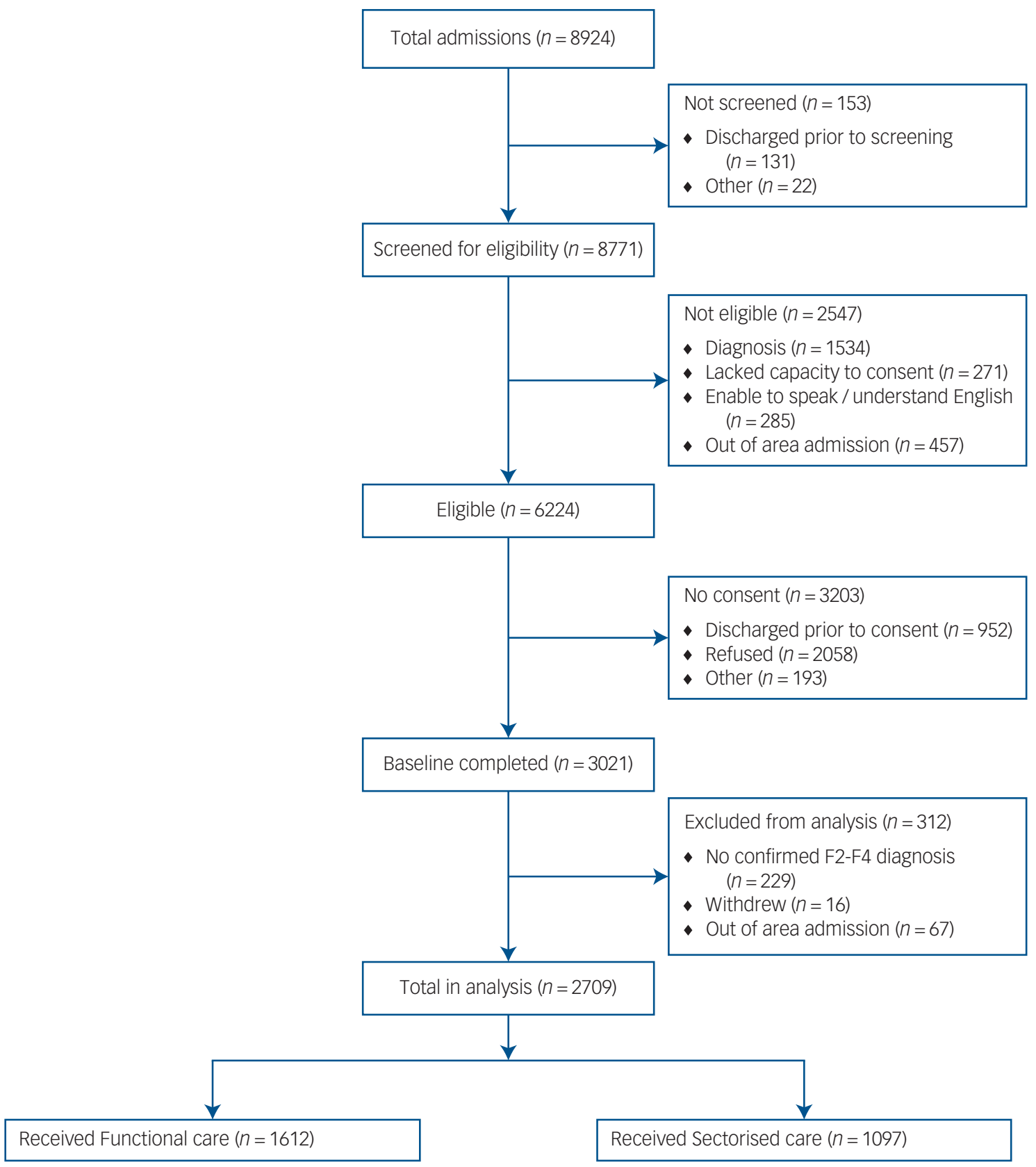

Fig. 1 Flow chart of participants through the study.

between patients in the two systems. All other sociodemographic variables did not differ. In total, 1155 patients $(42.6 \%)$ had a diagnosis of a psychotic disorder (F2), 1371 (50.6\%) a diagnosis of an affective disorder (F3) and 427 (15.8\%) had an anxiety/somatoform disorder (F4). There were no significant differences in the proportion of participants with psychotic or affective disorders between the two groups; however, the proportion of patients with a diagnosis of anxiety disorder was higher for those receiving sectorised care. Finally, more individuals receiving functional care were admitted involuntarily ( $45.5 \%$ v. $38.2 \%)$.

\section{Satisfaction with care}

On average patients were interviewed 5.9 days following admission (median 3 days, range 0-87 days; working days mean 4.1, median 2). The overall mean score for patient satisfaction with care across the CAT for the whole sample was 6.7 (s.d.=2.5). Mean satisfaction with care was 6.6 (s.d.=2.5) for functional and 7.1 (s. d. = 2.3) for sectorised care. At an individual-item level the mean difference between satisfactions scores for functional and sectorised care ranged from 0.4 to 0.8 , and was always favouring sectorised care.

Satisfaction with care was significantly greater for patients receiving sectorised care $(\beta=0.54,95 \%$ CI $0.35-0.73, P<0.001)$. This difference remained significantly higher when adjusting for clustering at the hospital level $(\beta=0.50,95 \%$ CI $0.17-0.82, P=$ $0.003)$ and all other covariates $(\beta=0.40,95 \%$ CI $0.06-0.74, P=$ $0.020)$. This represented a small effect size always favouring sectorised care $(d=0.12,0.09$ and 0.07 unadjusted, adjusted for clustering and fully adjusted model, respectively).

\section{LoS}

The mean LoS across the sample was 46.4 days (s.d. $=63.5)$. The mean LoS for functional care was 49.1 days (s.d. $=67.3$ ), whereas 
Table 1 Participant characteristics ${ }^{a}$

\begin{tabular}{|c|c|c|c|}
\hline & $\begin{array}{l}\text { Functional care } \\
\qquad(n=1612)\end{array}$ & $\begin{array}{l}\text { Sectorised care } \\
\quad(n=1097)\end{array}$ & $\begin{array}{l}\text { Total sample } \\
(n=2709)\end{array}$ \\
\hline Gender, male: $n(\%)$ & $954(59.1)$ & $630(57.4)$ & $1584(58.5)$ \\
\hline Age, years: mean (s.d.) & 40.7 (13.6) & 40.4 (13.2) & $40.6(13.5)$ \\
\hline $\begin{array}{l}\text { Marital status, } n(\%) \\
\text { Single } \\
\text { Married } \\
\text { Cohabiting/civil partnership } \\
\text { Separated } \\
\text { Divorced } \\
\text { Widowed } \\
\text { Not known }\end{array}$ & $\begin{array}{r}1003(62.2) \\
242(15.0) \\
69(4.3) \\
92(5.7) \\
151(9.4) \\
32(2.0) \\
14(0.9)\end{array}$ & $\begin{array}{r}657(59.9) \\
198(18.0) \\
47(4.3) \\
64(5.8) \\
97(8.8) \\
25(2.3) \\
5(0.4) \\
\end{array}$ & $\begin{array}{c}1660(61.3) \\
440(16.2) \\
116(4.3) \\
156(5.8) \\
248(9.2) \\
57(2.1) \\
19(0.7)\end{array}$ \\
\hline $\begin{array}{l}\text { Education, } n(\%)^{* *} \\
\text { Primary education or less } \\
\text { Secondary } \\
\text { Further education } \\
\text { Other } \\
\text { Not known }\end{array}$ & $\begin{array}{c}163(10.1) \\
585(36.3) \\
800(49.6) \\
45(2.8) \\
13(0.8)\end{array}$ & $\begin{array}{c}131(11.9) \\
461(42.0) \\
469(42.8) \\
21(1.9) \\
9(0.8)\end{array}$ & $\begin{array}{r}294(10.9) \\
1046(38.6) \\
1269(46.8) \\
66(2.4) \\
22(0.8)\end{array}$ \\
\hline $\begin{array}{l}\text { Accommodation } n(\%) \\
\text { Independent } \\
\text { Supported } \\
\text { Homeless } \\
\text { Other } \\
\text { Not known }\end{array}$ & $\begin{array}{r}1304(80.9) \\
149(9.2) \\
114(7.1) \\
34(2.1) \\
8(0.5)\end{array}$ & $\begin{array}{l}868(79.1) \\
111(10.1) \\
96(8.8) \\
12(1.1) \\
2(0.2)\end{array}$ & $\begin{array}{l}2172(80.2) \\
260(9.6) \\
210(7.8) \\
46(1.7) \\
10(0.4)\end{array}$ \\
\hline $\begin{array}{l}\text { Living situation, } n \text { (\%) } \\
\text { Alone } \\
\text { Family/partner } \\
\text { Friend } \\
\text { Shared accommodation }\end{array}$ & $\begin{array}{c}715(45.6) \\
684(43.7) \\
45(2.9) \\
122(7.8)\end{array}$ & $\begin{array}{c}487(45.8) \\
480(45.2) \\
36(3.4) \\
59(5.6)\end{array}$ & $\begin{array}{c}1202(45.7) \\
1164(44.3) \\
81(3.1) \\
181(6.9)\end{array}$ \\
\hline $\begin{array}{l}\text { Employment status, } n \text { (\%) } \\
\text { Full-time paid employed } \\
\text { Part-time paid employed } \\
\text { Voluntary } \\
\text { Unemployed } \\
\text { Housewife/husband } \\
\text { Student } \\
\text { Retired } \\
\text { Not known }\end{array}$ & $\begin{array}{l}257(15.9) \\
119(7.4) \\
44(2.7) \\
933(57.9) \\
32(2.0) \\
76(4.7) \\
96(6.0) \\
46(2.9)\end{array}$ & $\begin{array}{c}166(15.1) \\
78(7.1) \\
23(2.1) \\
689(62.8) \\
19(1.7) \\
34(3.1) \\
60(5.5) \\
25(2.3)\end{array}$ & $\begin{array}{c}423(15.6) \\
197(7.3) \\
67(2.5) \\
1622(59.9) \\
51(1.9) \\
110(4.1) \\
156(5.8) \\
71(2.6)\end{array}$ \\
\hline Receiving benefits, yes: $n(\%)$ & $1084(67.2)$ & $742(67.6)$ & $1826(67.4)$ \\
\hline Born in the UK, yes: $n(\%)^{*}$ & $1296(80.4)$ & $849(77.4)$ & $2145(79.2)$ \\
\hline $\begin{array}{l}\text { Diagnosis, } n(\%)^{b} \\
\text { Psychotic disorder (F2) } \\
\text { Affective disorder (F3) } \\
\text { Anxiety/somatic disorder (F4)** }\end{array}$ & $\begin{array}{l}683(42.4) \\
839(52.0) \\
192(11.9)\end{array}$ & $\begin{array}{l}472(43.0) \\
532(48.5) \\
235(21.4)\end{array}$ & $\begin{array}{r}1155(42.6) \\
1371(50.6) \\
427(15.8)\end{array}$ \\
\hline First admission, $n(\%)$ & $525(32.6)$ & $390(35.6)$ & $915(33.8)$ \\
\hline $\begin{array}{l}\text { Involuntary status at admission } \\
n(\%)^{* \star}\end{array}$ & $733(45.5)$ & $419(38.2)$ & $1152(42.5)$ \\
\hline CGI score at admission (mean, s.d.) & $4.4(1.3)$ & $4.4(1.4)$ & $4.4(1.4)$ \\
\hline
\end{tabular}

for sectorised care it was 42.3 days (s.d. $=57.3)$. Across the whole sample, the first, second (median) and third quartiles were 11, 25 and 52 days. In functional care they were 12, 26 and 55 days, and in sectorised care 10, 24 and 49 days, respectively. Quantile regression $(\mathrm{QR})$ indicated that patients receiving sectorised care had significantly shorter stays at all quartiles compared with those admitted in hospitals providing functional care (1st quartile $Q R$ coefficient $-2,95 \% \mathrm{CI}-3.17$ to $-0.28, P=0.023$, 2nd quartile $\mathrm{QR}$ coefficient $-2,95 \% \mathrm{CI}-4.01$ to $-0.01, P=0.051$ and 3rd quartile $\mathrm{QR}$ coefficient $-6,95 \% \mathrm{CI}-12.14$ to $-0.01, P=0.052)$. However, the difference was no longer statistically significant when clustering at the hospital level was accounted for (1st quartile QR coefficient -2, $95 \% \mathrm{CI}-6.29$ to $2.28, P=0.361$, 2nd quartile QR coefficient $-2,95 \%$ $\mathrm{CI}-6.99$ to $2.99, P=0.432$ and 3 rd quartile $\mathrm{QR}$ coefficient $-6,95 \% \mathrm{CI}$
-14.8 to $2.80, P=0.182)$ and after adjusting for clustering and covariates (1st QR coefficient $-0.75,95 \% \mathrm{CI}-5.18$ to $3.68, P=0.740$, 2nd QR coefficient $-0.40,95 \% \mathrm{CI}-5.33$ to $4.53, P=0.847$ and 3 rd QR coefficient $-0.29,95 \%$ CI -10.41 to $9.83, P=0.955)$. A KaplanMeier curve visually representing LoS for functional and sectorised care is shown in Supplementary Fig. 1 (available online at http://dx. doi.org/10.1192/bjp.2017.20).

To test the robustness of the quartile regression two sensitivity analyses were conducted, first because of the large sample size a mixed-effect linear regression was conducted using the means. Consistent with the above, patients receiving sectorised care had a significantly shorter admission by approximately 7 days compared with those admitted in hospitals providing functional care $(\beta=$ $-6.89,95 \% \mathrm{CI}-11.76$ to $-2.02, P<0.001)$. However, when adjusting 
for clustering, this difference was no longer statistically significant, and the number of days explained by the system of care was reduced to 4.9 days $(\beta=-4.89,95 \% \mathrm{CI}-13.34$ to $3.56, P=0.257)$. The number of days difference accounted for by the system of care was further reduced to 2 days when other covariates were added to the model $(\beta=-2.44,95 \% \mathrm{CI}-10.22$ to $5.34, P=0.539)$.

Second, as LoS data had a non-normal distribution, a further sensitivity analysis was conducted using a multilevel GLM with gamma distribution. As before, the results indicated a significant difference between functional and sectorised care (coefficient $-0.15,95 \% \mathrm{CI}-0.26$ to $-0.05, P=0.004$ ), which was no longer significant when adjusting for clustering at the hospital level (coefficient $-0.10,95 \% \mathrm{CI}-0.29$ to $0.09, P=0.292$ ) and other covariates (coefficient $-0.09,95 \% \mathrm{CI}-0.28$ to $0.10, P=0.335$ ).

\section{Discussion}

\section{Main findings}

Patients who receive sectorised mental healthcare are significantly more satisfied with their in-patient care compared with patients who receive functional care. This higher patient satisfaction was found in the first week after admission. The difference remained significant when the findings were adjusted for the influence of other factors such as locality of services, legal status, first or repeat admission and illness severity. Although patients in sectorised care stayed on average 7 days fewer than those in functional care, this difference was no longer significant once clustering by the hospital had been accounted for, suggesting factors aside from the organisation of care and patient characteristics have an impact on LoS.

\section{Strengths and limitations}

The data for the present study were collected in a natural experiment where the exposure of patients to either sectorised or functional care was outside the control of the investigators. ${ }^{5}$ A key limitation of existing studies is that they often assessed the effectiveness of a new system, where initial enthusiasm for newly introduced changes may influence the results. A previous review identified such a novelty effect. ${ }^{4}$ Consequently, a major strength of the present study is that we did not introduce a new system. Instead, sectorised or functional care was in routine practice for at least 1 year prior to the study. Thus, any systematic bias introduced by novelty effects was minimised. Within each site, allocation to sectorised or functional care was determined by geography and not influenced by the characteristics of the patient. Additionally, the study is one of the largest ever conducted with psychiatric in-patients within the UK. The large sample size, which is more than ten times larger than other studies directly comparing sectorised and functional care, enabled us to test for small differences, which at a national level may have wide implications. Finally, the study aimed to approach all potentially eligible participants within 2 working days after admission, with interviews conducted on average 6 days following admission. Often in-patient studies have assessed patients at least 1 or more weeks following admission, at the point of discharge or have collected satisfaction data retrospectively. This may bias the included sample, as individuals with a short admission may not be included. Research has also indicated that the appraisal of care changes during hospital admission. ${ }^{10}$

There are also a number of limitations. Most notably, this was not a randomised controlled trial (RCT), therefore, there may be non-random differences between the groups because of locality and the clinical and participant characteristics of the local area. Within the present study any patient characteristics shown to differ between the two systems were adjusted for. Furthermore, aside from the methodological problem of implementing a new system, one may argue that it would be practically impossible to randomly allocate a sufficient number of hospitals to sectorised and functional systems to conduct a formal RCT. Hospitals also varied in the types of acute wards provided. For instance, several hospitals included a triage or assessment ward, which impose a maximum stay before the patient is either discharged or transferred. However, existing evidence has suggested that the overall LoS for the hospital admission is not reduced by the use of these wards. ${ }^{14}$ Finally, the opt-in rate of $48.5 \%$ may make generalisability to all patients difficult, particularly if more unwell patients refused participation. However, there were no differences between the two types of care in the opt-in rates or various patient characteristics including illness severity, suggesting selection bias is unlikely to explain the results.

\section{Links with the existing evidence}

Qualitative studies assessing the experience of patients and clinicians have highlighted the benefits of both models of care, whereas quantitative studies directly addressing patient satisfaction tend to favour sectorised care. ${ }^{4}$ However, the majority of studies have investigated whether patients have a general preference for the same or different psychiatrists across settings, rather than investigating their actual experience. ${ }^{1}$ Additionally, studies have been limited by a number of factors including the implementation of a new system of care usually as part of the study, ${ }^{15}$ only focusing on patient satisfaction with the psychiatrist, ${ }^{16}$ and/or including only a small number of patients ranging from $41^{17}$ to $255 .^{1}$ The present study overcomes each of these limitations to provide more robust evidence.

One suggestion for the higher satisfaction of patients found in sectorised care is the impact of personal continuity. Participants in previous studies have highlighted how developing a positive therapeutic relationship ${ }^{18}$ and building trust ${ }^{19}$ may be harder to achieve if different clinicians are seen across settings. For instance, although not specific to psychiatrists, a higher turnover of mental health staff is associated with poorer patient outcomes ${ }^{20}$ and lower levels of satisfaction. ${ }^{21}$ Alternatively, the difference in satisfaction may be because of the attitude and behaviour of staff working in the different services. For example, there is some evidence to suggest that staff prefer the sectorised model of care. ${ }^{4}$ This preference and awareness of longer-term responsibility by the clinicians may influence their behaviour and hence have an impact on patient experience. However, the present study did not assess such potentially mediating factors. Future research should explore in depth the processes that mediate the impact of service organisations on patient satisfaction. This may include detailed organisational procedures, staff attitudes and behaviours as well as actual patient-clinician interactions during the in-patient admission.

Although the results show a clear finding regarding patient satisfaction, the comparison of LoS was not so clear-cut. The admission for patients receiving sectorised care was on average 7 days shorter than for those receiving functional care. The differences for the mean and median were not statistically significant once adjusting for other factors, including the hospital. This lack of statistical significance - despite the large sample size - reflected the large amount of variability in LoS. It is likely that LoS is influenced by a complex mix of factors above and beyond the model of care provided, including variables at the hospital level such as bed pressure, treatment practices, local clinical governance procedures and characteristics of the local area, as well as patient characteristics. In any case, one may conclude from the findings that functional care was not associated with shorter LoS.

The lack of an association of care organisation with LoS may be seen as consistent with previous research. Patient characteristics 
such as severity and chronicity of illness ${ }^{22,23}$ and a lack of social support $^{23,24}$ are all linked to longer LoS. Yet, good clinical care and the quality of services provided does not always result in reduced LoS once patient characteristics have been controlled for. ${ }^{23}$ Further research is needed to explore the individual patient factors and hospital factors, including the attitudes and behaviours of clinicians, which may be linked to shorter hospital admissions.

\section{Implications}

The study findings provide evidence for the ongoing debate about the pros and cons of a functional split of psychiatrist responsibility between in- and out-patient care. ${ }^{3}$ Despite the central importance of this question for patients and clinicians alike, decisions to change from one system to another - in England mostly from a sectorised to a functional one - have been made in the absence of any high-quality evidence.

In their debate article, Burns \& Baggeley outline the potential benefits of both sectorised care and a functional split in psychiatrist responsibility. ${ }^{3}$ Arguments for reconfiguring services have frequently included the stressful nature of in-patient wards, improving ward throughput, reducing bed occupancy and making the workload of the consultant psychiatrist more manageable. ${ }^{3}$ It has therefore been suggested that a functional split facilitates the management of in-patient wards and improves the quality of in-patient care. ${ }^{25}$ However, counterarguments for sectorised care point to the increased fragmentation within functional services and to increased patient and clinician satisfaction potentially as a result of the higher chance of developing a positive therapeutic relationship in sectorised systems. The present study provides the first sound evidence that patient satisfaction with in-patient treatment is greater in sectorised care. Thus, arguments for improved quality of care following a functional split are not supported. On the contrary one might argue that if patient satisfaction is a key indicator of quality of care, trusts may consider a transition back to a sectorised model which has been the case in one of the trusts (Bradford District Care Trust) included in the study.

The Francis inquiry report examining causes of the failings in care provided by Mid Staffordshire NHS Foundation Trust between 2005 and 2009, highlighted the importance of patient satisfaction to health service providers. ${ }^{26}$ Higher patient satisfaction with in-patient treatment is an important outcome in its own right, especially since satisfaction with in-patient care has deteriorated in recent years. ${ }^{27}$ Satisfaction is also an indicator for longer-term clinical outcomes. When assessing the outcomes of involuntary hospital admission, patient satisfaction with in-patient treatment was the only factor associated with both objective and subjective outcomes at 1 year follow up. In particular, higher initial treatment satisfaction was associated with lower rates of involuntary readmission and with a more positive appraisal of the admission retrospectively. ${ }^{9}$

In summary, the present study suggests that the current trend in the NHS of transforming services to functional care needs reconsidering. Sectorised care appears preferable in the interest of higher patient satisfaction with in-patient treatment.

\footnotetext{
V.J. Bird, PhD, D. Giacco, MD, PhD, Unit for Social and Community Psychiatry (WHO Collaborating Centre for Mental Health Service Development), Queen Mary University of London, London, UK; P. Nicaise, PhD, Institute of Health and Society IRSS, Université Catholique de Louvain, Bruxelles, Belgium; A. Pfennig, Dr.med., MSc, Department of Psychiatry and Psychotherapy, Carl Gustav Carus University Hospital, Technische Universität Dresden, Dresden, Germany; A. Lasalvia, MD, PhD, Section of Psychiatry, Department of Public Health and Community Medicine, University of Verona, Verona, Italy; M. Welbel, MA, Institute of Psychiatry and Neurology, Warsaw, Poland; S. Priebe FRCPsych, Unit for Social and Community Psychiatry (WHO Collaborating Centre for FRCPsych, Unit for Social and Community Psychiatry (WHO Collaborating Centre for
Mental Health Service Development), Queen Mary University of London, London, UK

Correspondence: Victoria Bird, Unit for Social and Community Psychiatry, Newham Centre for Mental health, London, E13 8SP, UK. Email: v.j.bird@qmul.ac.uk

First received 3 Feb 2017, final revision 23 Aug 2017, accepted 7 Nov 2017
}

\section{Funding}

This study was funded by the European Commission seventh Framework Programme. Grant agreement number is 602645 . The views expressed in this publication are those of the authors agreement number is 602645. The views expressed in this publication are those of the authors
and not necessarily those of the National Health Service, European Commission or Queen Mary University

\section{Supplementary material}

Supplementary material is available online at https://doi.org/10. 1192/bjp.2017.20.

\section{Acknowledgements}

The authors would like to grateful acknowledge the support of the funders, participants and wider COFI study group.

\section{References}

1 Begum M, Brown K, Pelosi A, Crabb J, McTaggart J, Mitchell C, et al. Survey of patients' view on functional split of consultant psychiatrists. BMC Health Serv Res 2013; 13: 362.

2 Burns T. The rise and fall of assertive community treatment? Int Rev Psychiatry 2010; 22: 130-7.

3 Burns T, Baggaley M. Splitting in-patient and out-patient responsibility does not improve patient care. Br J Psychiatry 2017; 210: 6-9.

4 Omer S, Priebe S, Giacco D. Continuity across inpatient and outpatient mental health care or specialisation of teams? A systematic review. Eur Psychiatry 2015; 30: 258-70.

5 Giacco D, Bird VJ, McCrone P, Lorant V, Nicaise P, Pfennig A, et al. Specialised teams or personal continuity across inpatient and outpatient mental healthcare? Study protocol for a natural experiment. BMJ Open 2015; 5: e008996.

6 World Health Organization. The ICD-10 Classification of Mental and Behavioural Disorders: Clinical Descriptions and Diagnostic Guidelines. WHO, 1992.

7 Priebe S, Gruyters T. Patients' and caregivers' initial assessments of treatment predicting hospitalisation. Schizophr Bull 1995; 21: 234-48.

8 Kallert TW, Priebe S, McCabe R, Kiejna A, Rymaszewska J, Nawka P, et al. Are day hospitals effective for acutely ill psychiatric patients? A European multicentre randomised controlled trial. J Clin Psych 2007; 68: 278-87.

9 Priebe S, Katsakou C, Amos T, Leese M, Morriss R, Rose D, et al. Patients' views and readmissions 1 year after involuntary hospitalisation. Br J Psychiatry 2009; 194: 49-54.

10 Katsakou C, Bowers L, Amos T, Morriss R, Rose D, Wykes T, et al. Coercion and treatment satisfaction among involuntary patients. Psychiat Serv 2010; 61: 286-92.

11 Priebe S, Jones G, McCabe R, Briscoe J, Wright D, Sleed M, et al. Effectiveness and costs of acute day hospital treatment compared with conventional inpatient care: randomised controlled trial. Br J Psychiatry 2006; 188: 243-9.

12 Richardson M, Katsakou C, Torres-González F, Onchev G, Kallert T, Priebe S. Factorial validity and measurement equivalence of the clients assessment of treatment scale for psychiatric inpatient care - a study in three european countries. Psychiat Res 2011; 118; 156-60.

13 Guy W. ECDEU Assessment Manual for Psychopharmacology. National Institute of Mental Health, 1976.

14 Williams P, Csipke E, Rose D, Koeser L, Mccrone P, Tulloch AD, et al. Efficacy of a triage system to reduce length of hospital stay. Br J Psychiatry 2014; 204: 480-5.

15 Lambert M, Bock T, Schottle D, Golks D, Meister K, Rietschel L, et al. Assertive community treatment as part of integrated care versus standard care: a 12month trial in patients with first- and multiple-episode schizophrenia spectrum disorders treated with quetiapine immediate release (ACCESS trial). J Clin Psychiatry 2010; 71: 1313-23.

16 Laugharne R, Pant M. Sector and functional models of consultant care: in patient satisfaction with psychiatrists. Psychiatrist 2012; 36: 254-6.

17 Le Bas J, King R, Block M. The impact of mental health service integration on systemic function: a staff perspective. Aust N Z J Psychiatry 1998; 32: 666-72.

18 Poremski D, Harris DW, Kahan D, Pauly D, Leszcz M, O'Campo P, et al. Improving continuity of care for frequent users of emergency departments: service user and provider perspectives. Gen Hosp Psychiatry 2016; 40: 55-9. 
19 Shan L, Li Y, Ding D, Wu Q, Liu C, Jiao M, et al. Patient Satisfaction with Hospital Inpatient Care: Effects of Trust, Medical Insurance and Perceived Quality of Care. PLOS One 2016; 11: e0164366.

20 Puntis SR, Rugkasa J, Burns T. The association between continuity of care and readmission to hospital in patients with severe psychosis. Soc Psychiatry Psychiatr Epidemiol 2016; 51: 1633-43.

21 Sanatinia R, Cowan V, Barnicot K, Zalewska K, Shiers D, Cooper JC, et al. Loss of relational continuity of care in schizophrenia: associations with patient satisfaction and quality of care. BJPsych Open 2016; 2: 318-22.

22 Nakanishi M, Niimura J, Tanoue M, Yamamura M, Hirata T, Asukai N. Association between length of hospital stay and implementation of discharge planning in acute psychiatric inpatients in Japan. Int J Ment Health Syst 2015; 9: 23.

23 Zhang J, Harvey C, Andrew C. Factors associated with length of stay and the risk of readmission in an acute psychiatric inpatient facility: a retrospective study. Aust N Z J Psychiatry 2011; 45: 578-85.
24 Gigantesco A, de Girolamo G, Santone G, Miglio R, Picardi A. Long-stay in shortstay inpatient facilities: risk factors and barriers to discharge. BMC Public Health 2009; 9: 306

25 Tulloch AD, Soper B, Gorzig A, Pettit S, Koeser L, et al. Management by Geographical Area or Management Specialised by Disorder? A Mixed-Methods Evaluation of the Effects of an Organisational Intervention on Secondary Mental Health Care for Common Mental Disorder. National Institute of Health Research, 2016.

26 Francis R. Report of the Mid Staffordshire NHS Foundation Trust Public Inquiry. The Stationery Office, 2013.

27 Csipke E, Williams P, Rose D, Koeser L, Mccrone P, Wykes T, et al. Following the Francis report: investigating patient experience of mental health in-patient care. Br J Psychiatry 2016; 209: 35-9.

\section{reflection}

\section{Bipolarity, creativity, stigma and authenticity in the work of Kay Redfield Jamison}

\section{John Cookson}

For many years, Kay Jamison has been the author I go to when advising patients diagnosed with bipolar disorder. Touched with Fire: Manic-depressive Illness and the Artistic Temperament (1993) is the first book I recommend. 'Touched' refers to mental imbalance and inspiration, and 'fire' refers to mania and passion. The book tells of numerous well-known figures who have been successful and outstanding despite (and possibly because of) their bipolar condition. Jamison has done as much as anyone to reduce the stigma of severe mental illness. I recommend her reflective accounts of her development as an academic clinical psychologist, struggling to come to terms with her own bipolar illness and need for treatment with a high dose of lithium, and with handling her career, marriages and bereavements (An Unquiet Mind: A Memoir of Moods and Madness (1996) and Nothing Was The Same: A Memoir (2009)).

Jamison is the co-writer, with Frederick Goodwin, of the definitive monograph on bipolar disorder, Manic-Depressive Illness: Bipolar Disorders and Recurrent Depression (1990 and 2007), a book of such scholarship, comprehensive understanding and enlightenment that it is unlikely to ever again be matched by two authors, considering the subsequent expansion in research.

Jamison has a zest for life and exuberance, but is also well acquainted with depression and suicidality, having begun to experience bipolar cycles at the age of 16 . She did not acknowledge these as symptoms of pathology, despite 3 years of graduate studies in clinical psychology, to include a final year at the Maudsley. She was diagnosed with psychotic mania when she was 17 years old, and again aged 29. If a patient with bipolar disorder declares that, after being told their diagnosis, they sought with a colleague to read everything they could find that had been written on the illness and its treatment, one might react with scepticism. To react so with Jamison would be wrong. She is a prolific reader ('three books a week') and her academic works are accompanied by appendices with fascinating expositions and references to other authors.

Touched with Fire presents evidence for the occurrence of manic and depressive periods in the lives of hundreds of famous people, notably poets, writers, composers and painters, and their relatives. It argues that the instability of mood in their illness, and their temperament, are integral to their productivity and originality. The argument is based partly on historical accounts of mood or temperament, and partly on detailed life charts and family trees. They include Robert Shuman, Alfred Lord Tennyson, Percy Bysshe Shelley, Samuel Taylor Coleridge, Virginia Woolf, Henry James, Ernest Hemingway, Vincent van Gogh, and a sketch of that most complete of manic-depressive creatives, with all the comorbidities, who gave his name to a temperament, George Gordon, Lord Byron. This connection needs to be documented because the stigma of mental illness prevents many individuals and their families from acknowledging it, whether they be successful, famous or not. Jamison's study on British artists, conducted during a sabbatical in Oxford and at St George's Hospital in 1982, contributed evidence for this association, which had also been explored by Nancy Andreasen in American writers. Jamison implies that the association might apply in other fields of artistic performance and leadership more broadly. Her own life is testament to this.

Before diagnosis, Jamison had 16 publications in the literature of personality types. Following her appointment in the Department of Psychiatry at the University of California, Los Angeles in 1974, she began publishing on bipolar disorder, and among her first publications were those with Frederick Goodwin in 1979 describing attitudes to lithium, and in 1980 on positive experiences reported by patients after affective episodes; by that time, she could draw on her own experiences for both topics. In 1983 she began work on Manic-Depressive IIIness, especially the sections on psychosocial aspects. By pointing to positive aspects of affective illness, Jamison's books help to dispel stigma and encourage people to speak about their experiences of it. They place the illness firmly in the domain of medical science.

Her achievement has not been easy. She received many exceedingly hostile letters from people with different views on the causes of mental illness, as well as from clinicians who encountered career blocks from stigma. However, she remains inspired by the sentiment of her most recent subject, Robert Lowell - 'Yet why not say what happened?'

Jamison discusses the ingredients of bipolarity that contribute to leadership, productivity and creativity. In her own case, the most impressive quality of her writing besides her extraordinary fluency and intelligence is its authenticity, born of personal experience and emotional courage. 